\title{
INFLUENCE OF GRAIN NUMBER ON GRAPHITE QUANTITATIVE TEXTURE STUDY
}

\author{
T.A. LYCHAGINA* and D.I. NIKOLAYEV \\ Frank Laboratory of Neutron Physics, Joint Institute for Nuclear Research, \\ Dubna, Moscow region, 141980, Russia
}

(Received 30 September 2003)

\begin{abstract}
The present work is devoted to the study of the grain number influence on the quantitative texture analysis and on the values of averaged elastic properties. Number of grains does not influence mathematical definition of orientation distribution function (ODF) (Bunge, H.J. (1982). Texture Analysis in Material Science. Butterworths, London; Matthies, S., Vinel, G.W. and Helming, K. (1987). Standard Distributions in Texture Analysis. Akademie-Verlag, Berlin.); nevertheless, intuitively clear that, averaging procedure implies a "large" number of grains to make sense. In the present work we applied the already suggested procedure (Lychagina, T.A. and Nikolayev, D.I. (2003). Phys. Stat. Sol. (a), 195(N2), 322-334.) for the case of hexagonal symmetry to evaluate the influence of the grain number in the sample on the calculated elastic properties. This procedure was carried out for graphite that is one of the widespread, applicable and highly anisotropic materials.
\end{abstract}

Keywords: Grain number; Graphite; Texture analysis

\section{INTRODUCTION}

A recently developed experimental technique of texture analysis based on the use of high-energy synchrotron radiation (Wcislak et al., 2002) made accessible the local texture measurements for sample series. The concept of grain statistics is very important in such investigations. One of the main aim of texture measurements is further determination of properties with taking texture into account. So to receive the reliable results we have to use special methods taking into account the limited number of grain in investigated volume as well.

One of the widely used methods to calculate physical properties is the averaging of single crystal properties with an orientation distribution function (ODF) (Bunge, 1982; Matthies et al., 1987). The method to evaluate the limitations for application of averaging procedures to real materials was already described in Lychagina et al. (2003). In that paper the influence of the grain statistics and the grain size distribution on averaging accuracy was estimated. For that it was proposed to simulate the grain microstructure according to Voronoi approach (Meijering, 1953; Gilbert, 1962;

*Corresponding author. Tel.: (709621)65972. Fax: (709621)65484. E-mail: lomteva@nf.jinr.ru 
Mullen et al., 1997) and three-dimensional random rotations distributed with known law (Roberts et al., 1984) simultaneously. In this work we extended such procedure to the highly anisotropic hexagonal material (graphite) and presented the numerical examples illustrating the influence of grain statistics and grain size distribution on the elastic constants of hexagonal textured materials as well as materials with completely random texture.

\section{THEORETICAL BACKGROUND}

We denote by $d V$ volume of all crystallites in a sample which possess an orientation $g$ within an orientation region $d g$, and by $V$ the total sample volume, then the ODF $f(g)$ is defined by Bunge (1982):

$$
f(g) d g=\frac{d V(g)}{V} .
$$

All rotations form the rotation group $S O(3)$. The ODF is defined on the rotation group $S O(3)$. The ODF defined by Bunge (1982) implies infinite number of orientations and we will call it "continuous" $\mathrm{ODF} f^{c}(g)$ with normalization property:

$$
\frac{1}{8 \pi^{2}} \int_{S O(3)} f^{c}(g) d g=1
$$

However, a real sample consists of a finite number of grains. If the number of grains in specimen is $N$, then the specimen is described by the "discrete" $\operatorname{ODF} f^{d}(g, N)$ in terms of $\delta$-functions on $S O(3)$ :

$$
f^{d}(g, N)=\frac{1}{V} \sum_{n=1}^{N} V_{n} \delta\left(g g_{n}^{-1}\right), \quad V=\sum_{n=1}^{N} V_{n}, \quad \frac{1}{8 \pi^{2}} \int_{S O(3)} \delta\left(g g_{n}^{-1}\right) d g=1
$$

The approach for calculation of mean physical properties, that does not make any speculations on the sample internal microstructure, is the arithmetic averaging of the single crystal properties $E$ for crystallites of orientation $g$ with the ODF $f(g)$ as a weighting function (Bunge, 1982; Matthies et al., 1987):

$$
\bar{E}^{a}=\frac{1}{8 \pi^{2}} \int_{S O(3)} E(g) f(g) d g .
$$

The averaging procedure (4) implies an infinite number of grains. However, any real sample or product possesses a finite volume and consequently a limited number of grains. Moreover, grains may be unevenly distributed in the considered volume. Both these aspects should be taken into account for the accurate application of (4). The usage of arithmetic averaging may be quite questionable due to the complete neglecting of the grain interaction. But for the statistical analysis presented in this article, the arithmetic mean drastically reduces the numerical effort and it is to be expected that the resulting critical grain numbers will be in the right order. 


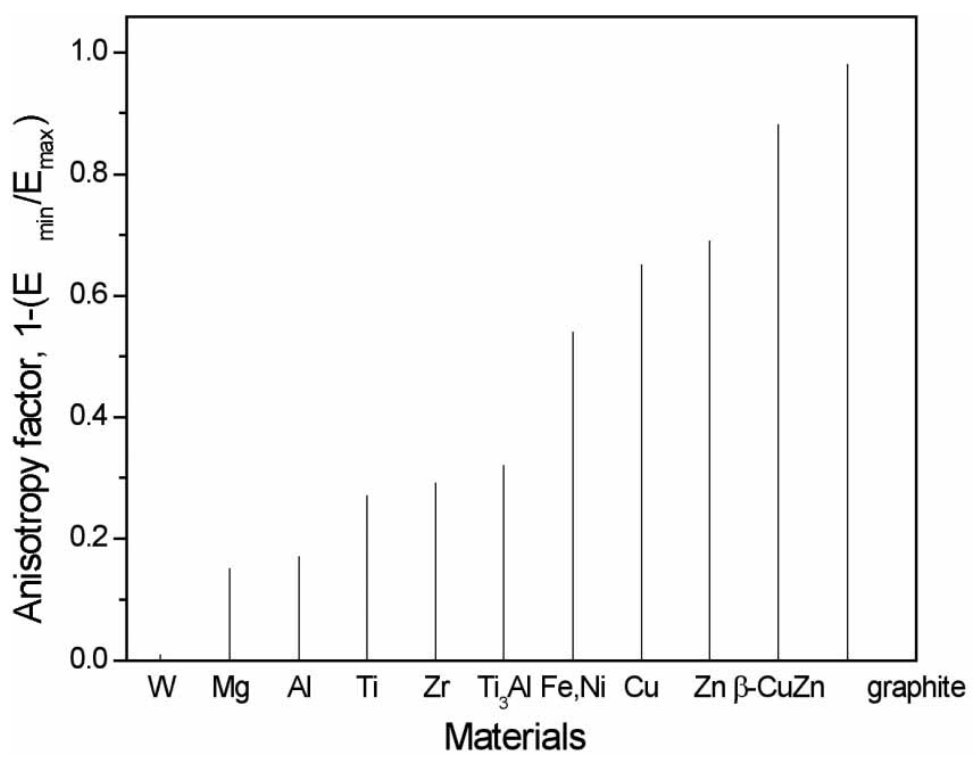

FIGURE 1 The elastic anisotropy of some materials.

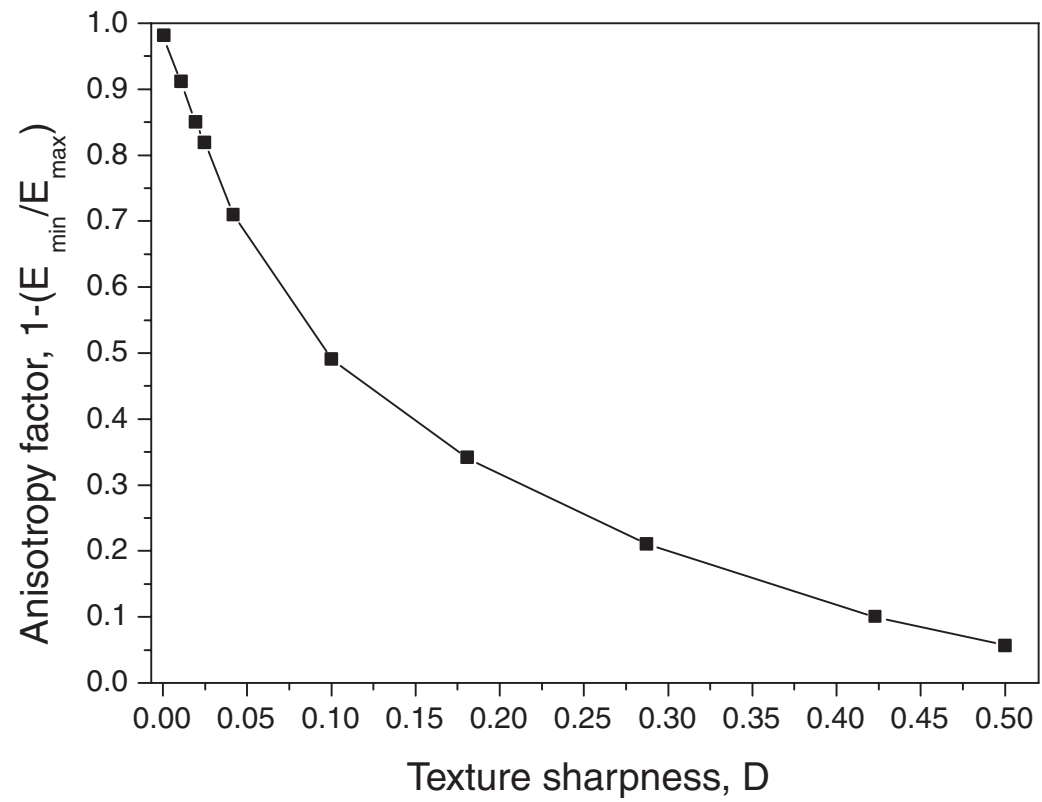

FIGURE 2 The dependence of anisotropy factor of graphite on texture sharpness.

All calculations carried out for highly anisotropic material graphite. The anisotropy factor is determined as $A=1-E_{\min } / E_{\max }$ and for graphite single crystal $A=0.98$ and this value is much more than that for copper, zinc and $\beta$-CuZn (Fig. 1). The dependence of anisotropy factor for textured polycrystalline graphite on texture sharpness is presented in Fig. 2. To receive this dependence texture is simulated by circular gaussian distribution (Savyolova, 1984) with center $g=\left\{0^{\circ}, 0^{\circ}, 0^{\circ}\right\}$ and width parameter 
$D$ controlling dispersion around of texture component maximum and describing texture sharpness.

\section{SIMULATIONS}

We simulated grain microstructure and an ensemble of individual orientations $g_{n} \in S O(3)$ for $N$ grains to investigate the influence of a grain number on the elastic constants. In order to generate microstructure pattern the cells (Voronoi) model was used (Meijering, 1953; Gilbert, 1962; Mullen et al., 1997). An ensemble of individual orientations $g_{n} \in S O(3)$ for $N$ grains was simulated according to the Roberts approach (Roberts et al., 1984).

To simulate grain microstructure we restricted ourselves to two-dimensional case, in other words we constructed plane Voronoi diagram. Let $S$ be a set of $N$ planar points $P_{i}$. The planar Voronoi diagram of $S$, as pictured in Fig. 3, is constructed in the following way. For each point $P_{i}$ of $S$, region $i$ is the set of all points of the plane which are closer to point $P_{i}$ than the other $N-1$ points of $S$. For application of the Voronoi model to generate of grain microstructures, $P_{i}$ represents the model location of the original nucleus or seed crystal from which the cell (grain) $i$ grew. One assumes for such application the following:

(i) the seeds for all crystals start growing at the same instant;

(ii) seeds grow at the same rate in all directions (i.e., as spheres); and

(iii) seeds stay fixed in space without pushing apart as they grow into contact.

To simulate planar Voronoi diagram we used the algorithm based on a three-dimensional convex hull (Brown, 1979; Sugihara, 2000). In Fig. 3(b)-(d), the examples of microstructure or planar Voronoi diagrams constructed for $N=3000$ random uniformly distributed input points and for $N=3000$ input points random distributed according to normal and exponential laws are given. The areas of Voronoi cells have been normalized so that the squares presented in Fig. 3(b)-(d) have area $1 \mathrm{~cm}^{2}$. Based on planar grain microstructure, grain size distribution can be calculated. These distributions for microstructures constructed for $N=5000$ input points randomly distributed according to different laws were presented in Lychagina et al. (2003).

To simulate an ensemble of individual orientations $g_{n} \in S O(3)$ for $N$ grains we assumed in compliance with Roberts approach that ensemble $g_{n}$ is distributed according to the Brownian motion law on $S O(3)$. If one considers a sequence of $t$ rotations through a fixed angle $\Lambda$ about a randomly oriented axis with random direction, and Brownian motion starts at $g=\left\{0^{\circ}, 0^{\circ}, 0^{\circ}\right\}(g \in S O(3))$, then the distribution density $\rho_{t}(\boldsymbol{r}), \boldsymbol{r} \in S^{3} \subset R^{4}$ after $t$ rotations at special conditions $t \rightarrow \infty, \Lambda \rightarrow 0, \Lambda^{2} t \rightarrow$ const can be written as follows (Roberts et al., 1984):

$$
\rho(\boldsymbol{r})=\frac{1}{2 \pi^{2}} \sum_{l=0}^{\infty}(2 l+1) \exp \{-l(l+1) D\} \frac{\sin ((2 l+1)(\omega / 2))}{\sin (\omega / 2)}, \quad D=\frac{\Lambda^{2} t}{6} .
$$

The distribution corresponding to (5) on the sphere $S^{2} \subset R^{3}$ is (Luzin, 1999):

$$
\rho(\boldsymbol{y})=\frac{1}{4 \pi} \sum_{l=0}^{\infty}(2 l+1) \exp \{-l(l+1) D\} P_{l}(\cos \beta), \quad \boldsymbol{y}=(\beta, \varphi) \in S^{2},
$$


(a)

a)

(c)

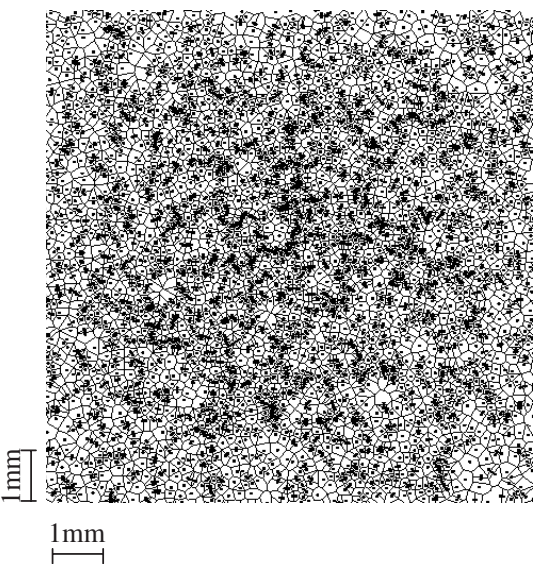

(b)

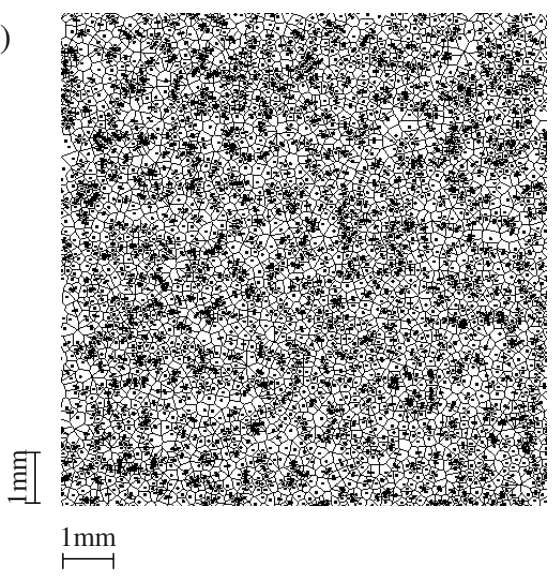

(d)

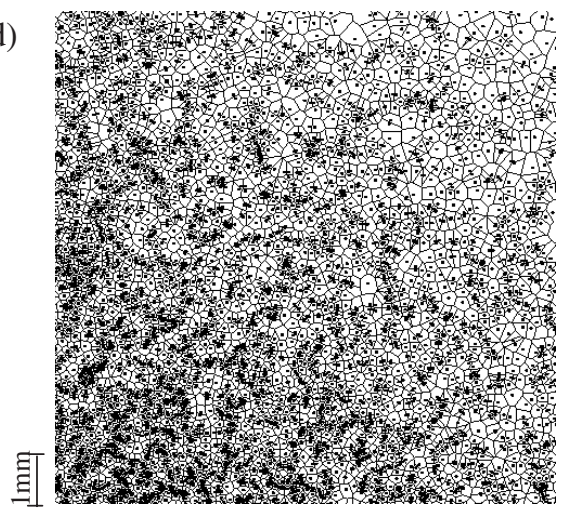

$1 \mathrm{~mm}$

FIGURE 3 Voronoi 2-dimensional diagrams - microstructures constructed for: (a) $N=600$ random uniform distributed input points; (b) for $N=3000$ random uniform distributed input points; (c) for $N=3000$ input points distributed according to normal law; (d) for $N=3000$ input points distributed according to exponential law. The squares presented in Fig. 3(b)-(d) have area $1 \mathrm{~cm}^{2}$. The center of normal distribution is in the center of Fig. 3(c), where is the maximal number of grains; the center of exponential distribution is in the left lower corner of Fig. 3(d) where there is maximal number of grains.

where $P_{l}(\cos \beta)$ are Legendre polynomials, $D$ is the parameter connected to the strength of the texture. To generate the ensemble of $g_{n}$ distributed with density (5) choose $\Lambda_{0} \ll 1$ and $t_{0} \gg 1$ so that $D=\Lambda_{0}^{2} t_{0} / 6$ is valid. Besides, an appropriate symmetrization (Matthies et al., 1987) is required to obtain the actual ODF and PFs. After subjecting the initial orientation $g=\left\{0^{\circ}, 0^{\circ}, 0^{\circ}\right\}$ to a series of rotations through a fixed angle $\Lambda$ around the random axis we obtain the ensemble of $g_{n}$ distributed with density (5), which is the texture component with sharpness $D$. To evaluate the quality of the experimental PFs the $R$-factor is introduced (Luzin, 1999):

$$
R \approx \frac{1}{M} \sum_{m=1}^{M} R P\left(\varepsilon, P_{\boldsymbol{h}}^{c}(\boldsymbol{y}), P_{\boldsymbol{h}}^{e, m}(\boldsymbol{y}, N)\right),
$$


$\begin{array}{llll}\text { "continuous" } \quad \mathrm{N}=10000 \quad \mathrm{~N}=20000 & \mathrm{~N}=50000 & \mathrm{~N}=100000\end{array}$

(001)
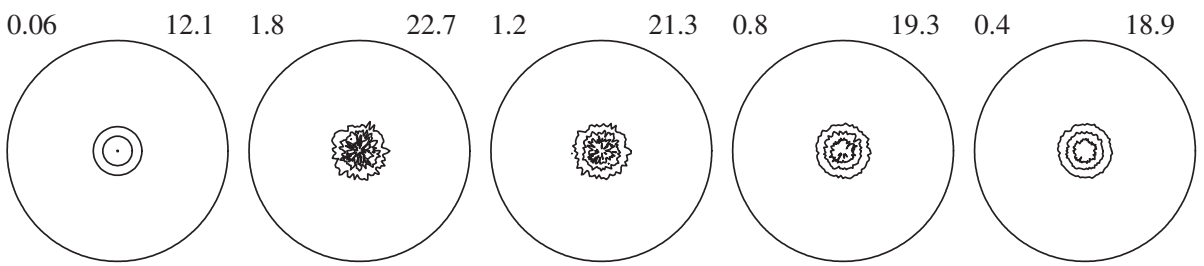

(100)

$$
R=44 \%
$$

$R=36.5 \%$

$R=25.0 \%$

$R=12.5 \%$
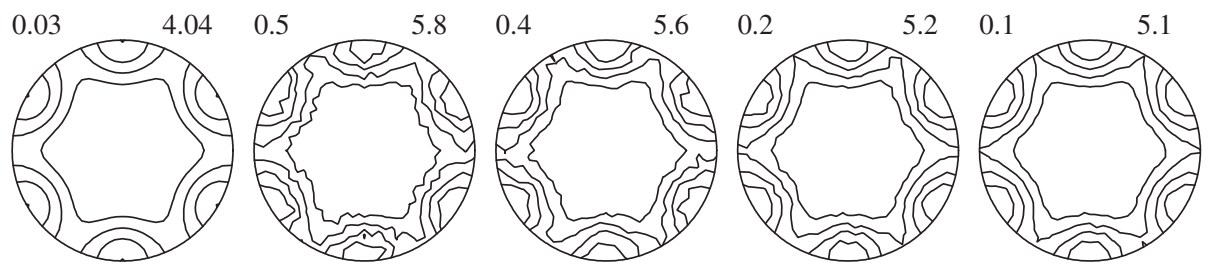

$R=41.3 \%$

$R=33.1 \%$

$R=22.4 \%$

$R=11 \%$

$(101)$
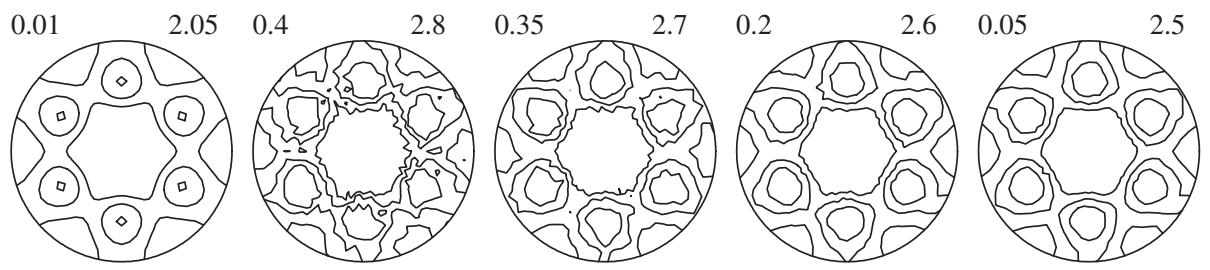

$R=38.7 \%$

$R=28.3 \%$

$R=17 \%$

$R=10.3 \%$

FIGURE 4 The individual realization $(M=1)$ of pole figures (stereographic projection) for hexagonal material simulated on grid with step $5^{\circ}$ with the texture model $g=\left\{0^{\circ}, 0^{\circ}, 0^{\circ}\right\}, D \approx 0.042\left(H W H M \approx 19.5^{\circ}\right)$ : first column is "continuous" PFs, last columns are "discrete" PFs simulated for samples with different number of grains. Contours are for PF (001) 4, 8, $12 \mathrm{mrd}$; for PF (100) 1, 2, 3, 4 mrd; for PF (101) 1, 1.5, 2 mrd.

where $P_{h}^{e, m}(\boldsymbol{y}, N)$ is the $m$ th independent realization of the simulated experimental PF, $M$ is the number of independent realizations, $P_{h}^{c}(\boldsymbol{y})$ is the known distribution of "continuous" PF. The RP factor can be determined as following (Matthies et al., 1988):

$$
R P=\sum_{j=1}^{J} 100 \% \Theta\left[\varepsilon, P_{h}\left(\boldsymbol{y}_{j}\right)\right] r_{j} / \sum_{j=1}^{J} \Theta\left[\varepsilon, P_{\boldsymbol{h}}\left(\boldsymbol{y}_{j}\right)\right],
$$

where

$$
r_{j}=\left|P_{\boldsymbol{h}}^{c}\left(\boldsymbol{y}_{j}\right)-P_{\boldsymbol{h}}^{e, m}\left(\boldsymbol{y}_{j}\right)\right| / P_{\boldsymbol{h}}^{c}\left(\boldsymbol{y}_{j}\right), \quad \Theta(\varepsilon, x)= \begin{cases}0, & x \leq \varepsilon \\ 1, & x>\varepsilon\end{cases}
$$

In Fig. 4 the individual realization $(M=1)$ of PFs simulated for hexagonal material with different grain number and a single component texture model are given. The 
$D=0.042$

(001)

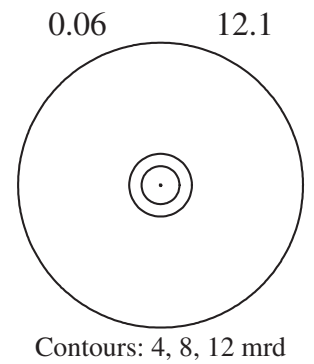

(100)

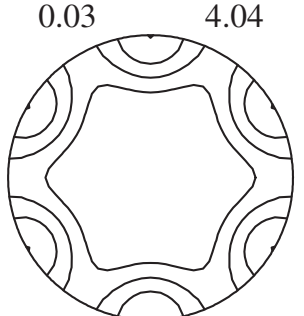

Contours: 1, 2, 3, 4 mrd

(101)

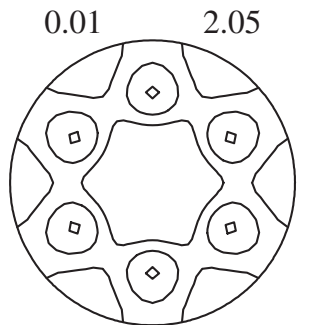

Contours: 1, 1.5, $2 \mathrm{mrd}$
$D=0.0192$
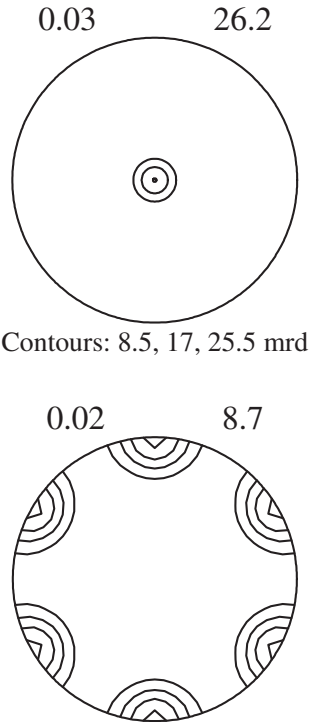

Contours: 2, 4, 6, 8 mrd

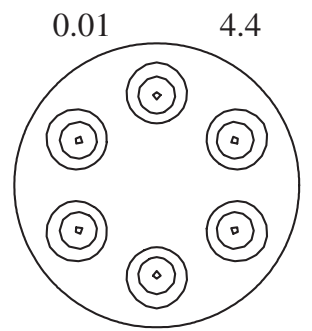

Contours: 1.4, 2.8, $4.2 \mathrm{mrd}$

FIGURE 5 The "continuous" pole figures of hexagonal material with different width parameter $D$. The "discrete" pole figures reveal the same dependence on texture sharpness.

center of the component is $g=\left\{0^{\circ}, 0^{\circ}, 0^{\circ}\right\}$ and width parameter $D \approx 0.042$ was determined using $\Lambda_{0}=0.025$ and $t_{0}=400$. This corresponds to $H W H M \approx 19.5^{\circ}$ of the texture component. The values of $D$ and $H W H M$ are obtained numerically. As can be seen from Fig. 4, increasing the number of grains minimizes the $R$-factor and makes simulated PFs closer to "continuous" PFs. Figure 5 shows the dependence between pole figures and texture sharpness, controlled by the width parameter $D$.

\section{RESULTS}

We calculated elastic properties for the set of "samples" of graphite with different number of grains. The single-crystal data were taken from Hellwege (1979) (see Table I).

To underline the importance of grain size distributions for the modeling, the results for models with equal volume grains are presented first. 
TABLE I Single crystal compliance data of graphite used in calculations $\left(\times 10^{-2} \mathrm{GPa}^{-1}\right)$

\begin{tabular}{lcccc}
\hline$S_{11}$ & $S_{12}$ & $S_{13}$ & $S_{33}$ & $S_{44}$ \\
\hline 0.11 & -0.0046 & -0.249 & 3.32 & 43.5 \\
\hline
\end{tabular}

The properties with "discrete" ODF (3) can be found in the framework of an equal grain model $\left(V_{n}=V_{0}=V / N, n=1, \ldots, N\right)$ as follows:

$$
E_{i j k l}(N)=\frac{1}{8 \pi^{2}} \int_{S O(3)} E_{i j k l}(g) f^{d}(g, N) d g=\frac{1}{N} \sum_{n=1}^{N} E_{i j k l}\left(g_{n}\right),
$$

where $E_{i j k l}\left(g_{n}\right)$ is the property of grain $n$. Considering the more realistic case where the grain volumes are distributed according to some law, the properties with "discrete" ODF (3) can be found as follows:

$$
E_{i j k l}(N)=\frac{1}{8 \pi^{2}} \int_{S O(3)} E_{i j k l}(g) f^{d}(g, N) d g=\frac{1}{V} \sum_{n=1}^{N} E_{i j k l}\left(g_{n}\right) V_{n},
$$

where $V$ is the sample size, $V_{n}$ is the $n$th grain size, $E_{i j k l}\left(g_{n}\right)$ is the property of grain $n$.

Ensembles of individual orientations having gaussian distributions with center $g=\left\{0^{\circ}, 0^{\circ}, 0^{\circ}\right\}$, width parameter $D \approx 0.042\left(H W H M \approx 19.5^{\circ}, \Lambda_{0}=0.025, t_{0}=400\right)$ and $g=\left\{0^{\circ}, 0^{\circ}, 0^{\circ}\right\}, D \approx 0.0192\left(H W H M \approx 13.2^{\circ}, \Lambda_{0}=0.012, t_{0}=800\right)$ were simulated.

Then the Young's module was determined assuming equal grain size as follows:

$$
E_{1111}(N)=\frac{1}{M} \sum_{m=1}^{M} E_{1111}^{m}(N)
$$

where

$$
E_{1111}^{m}(N)=\frac{1}{S_{1111}^{m}(N)}, \quad S_{1111}^{m}(N)=\frac{1}{N} \sum_{n=1}^{N} S_{1111}\left(g_{n}^{m}\right),
$$

$S_{1111}(g)$ is the component of compliance tensor given in sample coordinate system, $E_{111}^{m}(N)$ is the result of the $m$ th statistical realization of the algorithm to simulate a set of individual orientations $g_{n}^{m}, M$ is the number of independent realizations of this algorithm.

In case of grain volume distributions Young's module was determined as an averaging of $M$ independent statistical realizations of the algorithm to simulate a set of individual orientations, and the algorithm for Voronoi diagram constructing. The result of $m$ th realization in this case was determined as follows:

$$
E_{1111}^{m}(N)=\frac{1}{S_{1111}^{m}(N)}, \quad S_{1111}^{m}(N)=\frac{1}{V} \sum_{n=1}^{N} S_{i j k l}\left(g_{n}^{m}\right) V_{n}^{m},
$$


where $V$ is sample size, $V_{n}^{m}$ is the $n$th grain size in $m$ th realization. The relative error of the Young's module calculation was determined as a function of a number of grains in a "sample":

$$
\delta=\frac{\left|E_{1111}(N)-E_{1111}^{R}\right|}{\min \left(E_{1111}(N), E_{1111}^{R}\right)},
$$

where $E_{1111}^{R}$ is Young's module calculated by Reuss approximation (i.e. with assumption $N \rightarrow \infty)$ :

$$
E_{1111}^{R}=\frac{1}{\left\langle S_{1111}\right\rangle}, \quad\left\langle S_{1111}\right\rangle=\frac{1}{8 \pi^{2}} \int_{0}^{2 \pi} \int_{0}^{\pi} \int_{0}^{2 \pi} S_{1111}(\alpha, \beta, \gamma) f^{c}(\alpha, \beta, \gamma) \sin \beta d \alpha d \beta d \gamma
$$

where $S_{1111}(\alpha, \beta, \gamma)$ is the component of compliance tensor given in sample coordinate system, $f^{c}(\alpha, \beta, \gamma)$ is a "continuous" ODF.

The relative error $\delta$ of Young's module calculation as a function of the grain number has been determined with and without taking the grain size distribution into account. The results of Young's module calculations for graphite with the texture models already mentioned above, including completely random texture, are presented in Fig. 6. In our previous investigation (Lychagina et al., 2003) we established that the results of Young's module calculations weakly depend on distribution of input points (grain seeds). So to receive results presented in Fig. 6, the grain size distribution obtained for random uniform distribution of input points was used. As can be seen from this figure, the

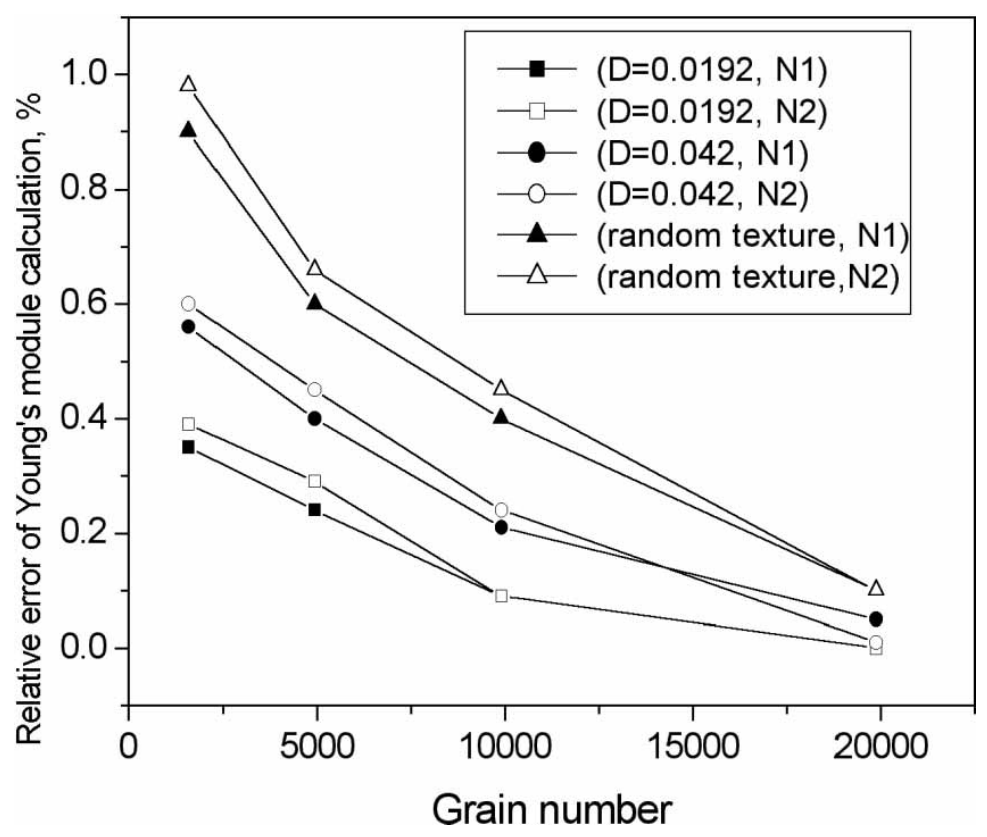

FIGURE 6 The relative error of Young's module calculation for graphite evaluated for completely random texture and for two texture models $g=\left\{0^{\circ}, 0^{\circ}, 0^{\circ}\right\}(D \approx 0.042, D \approx 0.0192)$ as a function of grain number without (calculation $N 1$ ) and with (calculation $N 2$ ) taking grain size distribution into account. 
relative error $\delta$ decreases with increasing the number of grains in the "sample." Moreover, the sharper the texture of material the weaker is the dependence of elastic properties on the number of grains. Besides, the influence of the grain size distribution increases the relative error of Young's module calculation. We adopted the following numerical criterion for the valuable difference between the calculations with and without taking this distribution into account $\delta^{c}>0.01 \%$. With increasing a number of grains $N$ in sample the difference between errors of Young's module calculation with and without taking grain size distribution into account decreases and can be neglected for $N \approx 15000$ in case of texture model with $D \approx 0.042$. For sharper texture this difference decreases as well and can be neglected for smaller number of grains $(N \approx 10000)$. For completely random texture the corresponding number of grains is $N \approx 20000$.

\section{SUMMARY}

The algorithm to evaluate limitations for arithmetic averaging technique is applied for graphite that is the hexagonal material with high anisotropy factor. These limits were determined for different texture models. The minimum number of grains for conventional averaging is about 10000 in case of graphite for one of the considered texture model and 20000 for completely random texture. Taking into account the influence of grain size distributions increases the error of elastic property calculation.

\section{References}

Brown, K.Q. (1979). Information Processing Letters, 9(5), 223-228.

Bunge, H.J. (1982). Texture Analysis in Material Science. Butterworths, London.

Gilbert E.N. (1962). Ann. Math. Statist., 33, 958-972.

Hellwege, K.-H. (1979). Numerical data and functional relationships in science and technology. Group III. Crystal and Solid State Physics, Vol. 11. Springer-Verlag, Berlin.

Luzin, V.V. (1999). Textures and Microstructures, 33, 343-355.

Lychagina, T.A. and Nikolayev, D.I. (2003). Phys. Stat. Sol. (a), 195(N2), 322-334.

Matthies, S., Vinel, G.W. and Helming, K. (1987). Standard Distributions in Texture Analysis. AkademieVerlag, Berlin.

Matthies, S., Wenk, H.R. and Vinel, G. (1988). J. Appl. Cryst., 21, 285-304.

Meijering, J.L. (1953). Philips Res. Rep., 8, 270-290.

Mullen, R., Ballarini, R., Yin, Y. and Heuer, A.N. (1997). Acta Materialia., 45(N6), 2247-2255.

Roberts, P.H. and Winch, D.E. (1984). Adv. Appl. Prob., 16, 638-655.

Savyolova, T.I. (1984). Zavodskaya Laboratoriya, 50, 48-52 (Russian).

Sugihara, K. (2000). Theoretical Computer Science, 235, 325-337.

Wcislak, L., Klein, H., Bunge, H.J., Garbe, U., Tschentscher T. and Schneider, J.R. (2002). J. Appl. Cryst., $35,82-95$. 